University of Louisville

ThinkIR: The University of Louisville's Institutional Repository

Electronic Theses and Dissertations

8-2012

\title{
Effectiveness of fluoride containing bonding resins in preventing demineralization of enamel during orthodontic treatment.
}

Robin J. Choi

University of Louisville

Follow this and additional works at: https://ir.library.louisville.edu/etd

\section{Recommended Citation}

Choi, Robin J., "Effectiveness of fluoride containing bonding resins in preventing demineralization of enamel during orthodontic treatment." (2012). Electronic Theses and Dissertations. Paper 246.

https://doi.org/10.18297/etd/246

This Master's Thesis is brought to you for free and open access by ThinkIR: The University of Louisville's Institutional Repository. It has been accepted for inclusion in Electronic Theses and Dissertations by an authorized administrator of ThinkIR: The University of Louisville's Institutional Repository. This title appears here courtesy of the author, who has retained all other copyrights. For more information, please contact thinkir@louisville.edu. 


\title{
EFFECTIVENESS OF FLUORIDE CONTAINING BONDING RESINS IN PREVENTING DEMINERALIZATION OF ENAMEL DURING ORTHODONTIC TREATMENT
}

\author{
By \\ Robin J. Choi \\ BS, University of Maryland at College Park, 2003 \\ DDS, University of Maryland at Baltimore, 2010
}

\begin{abstract}
A Thesis
Submitted to the Faculty of the University of Louisville School of Dentistry in Partial Fulfillment of the Requirements for the Degree of
\end{abstract}

Masters in Oral Biology

Department of Orthodontics

School of Dentistry

University of Louisville

Louisville, Kentucky

August 2012 


\section{EFFECTIVENESS OF FLUORIDE CONTAINING BONDING RESINS IN \\ PREVENTING DEMINERALIZATION OF ENAMEL DURING \\ ORTHODONTIC TREATMENT}

$$
\text { By }
$$

Robin J. Choi

BS, University of Maryland at College Park, 2003

DDS, University of Maryland at Baltimore, 2010

A Thesis Approved on

June 7, 2012

by the following Thesis Committee:

Sunita Chandiramani, DMD, MS

Thesis Director

David Tasman, DMD

Anibal Silveira, DDS 


\section{DEDICATION}

I lovingly dedicate this thesis to my parents to whom I owe every success in life. 


\section{ACKNOWLEDGMENTS}

I would like to thank my committee members, Dr. Sunita Chandiramani, Dr. David Tasman, and Dr. Anibal Silveira, for all their support throughout this process. I would also like to extend a special note of thanks to Mr. Alex Cambon for his enthusiasm and for his efforts in providing a better understanding of the data by way of a thorough statistical analysis and report. 


\title{
ABSTRACT \\ EFFECTIVENESS OF FLUORIDE CONTAINING BONDING RESINS IN PREVENTING DEMINERALIZATION OF ENAMEL DURING ORTHODONTIC TREATMENT
}

\author{
Robin J. Choi
}

June 7, 2012

Introduction: Although orthodontics can correct a variety of dental and skeletal malocclusions, research has shown an increased risk of enamel demineralization leading to white spot lesions (WSLs). The purpose of this study was to compare the effectiveness of Pro Seal and Opal Seal, two fluoridated bonding agents, to Assure, a nonfluoridated bonding agent, in preventing demineralization during orthodontic treatment. Methods: 26 patients beginning comprehensive orthodontic treatment were divided into 3 groups. 9 patients were bonded with Assure (control), 9 patients were bonded with Pro Seal, and 8 patients were bonded with Opal Seal. Pretreatment and six-month progress photographs were used to calculate demineralization rates, and statistical analysis was performed to determine if any bonding agent is better at preventing WSLs. Results: There was no statistically significant difference in demineralization rates between the three bonding agents; however, descriptive statistics suggest that Pro Seal and Opal Seal may prevent demineralization more effectively than Assure. 


\section{TABLE OF CONTENTS}

PAGE

ACKNOWLEDGEMENTS ........................................................

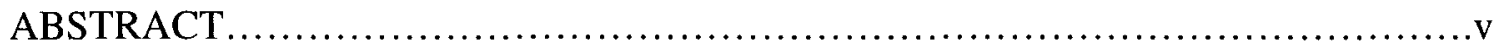

\section{CHAPTER}

I. INTRODUCTION

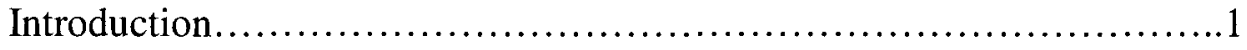

Literature Review..................................................

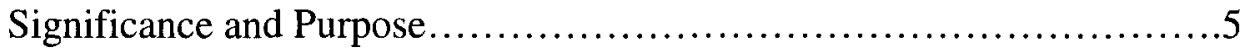

Hypotheses...............................................................

II. METHODS AND MATERIALS

Study Design and Sample.........................................6

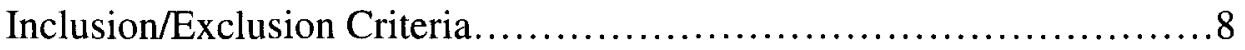

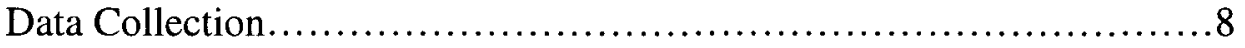

Statistical Analysis...................................................

\section{RESULTS}

Data Description.................................................. 10

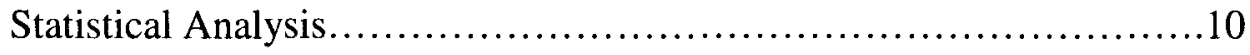

Results.......................................................

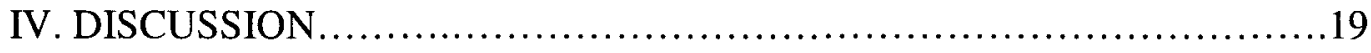

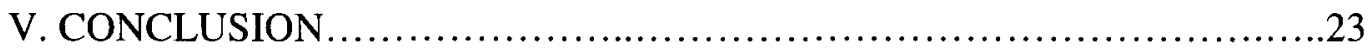

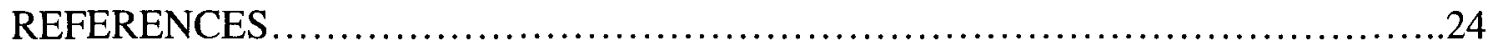

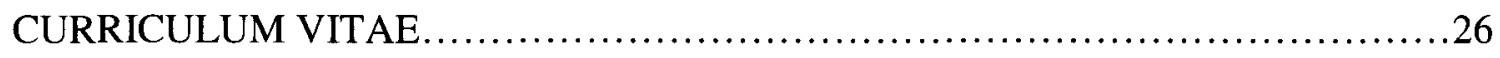




\section{CHAPTER I}

\section{INTRODUCTION}

\section{Introduction}

Although orthodontics has the ability to correct a variety of dental and skeletal malocclusions, increased risk of enamel demineralization around brackets and gingival margins during treatment has been a widely reported downside. Demineralization manifests itself as white spot lesions (WSLs) on the surfaces of teeth. WSLs are defined as "the first sign of a caries lesion on enamel that can be detected with the naked eye" (Shungin et al, 2010). Proper oral hygiene around bonded brackets and archwires is challenging for patients, oftentimes leading to decreased patient motivation and increased incidence of plaque buildup between appointments (Farhadian et al, 2008). Orthodontic appliances frequently serve as extra retention sites for plaque in the oral cavity (Chang et al, 1997), and the likelihood of increased plaque accumulation raises the risk of demineralization, which is sometimes evident as early as within a month of initial bonding (Heintz and Georg, 1999). Decalcification rates as high as $96 \%$ have been reported in the literature (Mitchell, 1992). As such, preventive measures during orthodontic treatment to counteract the increased risk of demineralization are of paramount importance. 


\section{Literature Review}

In addition to serving as plaque retentive sites, orthodontic appliances may hinder the natural ability of the tongue to remove food and lead to stagnation areas, resulting in lactobacillus counts that are five times higher than normal in orthodontic patients (Chang et al, 1997). Maxillary lateral incisors along with mandibular canines and mandibular premolars are the teeth most susceptible to demineralization, with the maxillary anterior teeth being particularly susceptible because it is in an area of decreased salivary flow and increased carbohydrate exposure (Chang et al, 1997). Interestingly, a close positive association also has been found between clinical attachment level, the sum of gingival recession and sulcus probing depth, and WSLs (Lovrov et al, 2007).

Fluoride has long been advocated in dental care for its ability to prevent and arrest demineralization, and topical fluoride use has been shown to be helpful during orthodontic treatment (Chadwick et al, 2005; Behnan et al, 2010). Studies have shown that fluoride varnish at regular intervals decreases demineralization occurrence, but requires repeated application and chair time, which may prove to be problematic. One particular study found that a single high dose application of fluoride varnish at the beginning of orthodontic treatment could reduce WSL depths by $40 \%$ during the first three months of treatment (Farhadian et al, 2008). Sodium fluoride mouthwash use during treatment has also been found to be effective; unfortunately, less than $12 \%$ of patients will exhibit excellent compliance with the mouthwash regimen (Paschos et al, 2009), rendering its benefits minimal. In an effort to take patient compliance out of the equation, a 1996 study with unfilled sealants suggested that the application of a lightcured sealant on teeth with orthodontic brackets may result in drastically less 
demineralization compared with teeth left unsealed when subjected to a demineralization environment (Frazier et al, 1996). Light-cured sealants have been found to be more successful in prevention of decalcification when compared to chemical-cured sealants due to the lack of the oxygen inhibition of polymerization that occurs with chemicalcured sealants (Shinaishin et al, 2011).

Many orthodontic bonding materials, including bonding adhesives, have incorporated fluoride in an attempt to decrease the incidence of demineralization caused, in part, iatrogenically by fixed orthodontic appliances. A study in 1996 by Trimpeneers and Dermaut found that there was no significant difference between Orthon, a light-cured fluoride releasing bracket adhesive, and a control non-fluoridated adhesive. A 2010 systematic review published in the American Journal of Orthodontics and Dentofacial Orthopedics (AJODO) came to the conclusion that only glass ionomer adhesives have a statistically significant effect on lowering decalcification rates, but traditional glass ionomer cements have low bond strengths and are therefore not recommended for bonding orthodontic brackets (Rogers et al, 2010).

Orthodontic bonding agents and sealants have also recently incorporated fluoride; however, research on their efficacy has been somewhat inconclusive. A pilot study by Benham et al found that the use of sealants on teeth with fixed orthodontic appliances reduced WSLs by 3.8 times (Benham et al, 2009). Pro Seal (Reliance Orthodontic Products) and Opal Seal (Ultradent) are two such fluoridated sealant bonding agents on the market today, and they both purport to protect teeth that have been bonded with orthodontic brackets. Several studies have reported conflicting results on the efficacy of Pro Seal in preventing decalcification, but to date there appears to be only one published 
study on Opal Seal in which Hess et al showed in vitro that Opal Seal reduced decalcification rates by 5\%, a statistically significant amount (Hess et al, 2011).

A 2009 study reported that although application of Pro Seal and Clearfil Protect Bond (Kuraray Medical), both fluoride releasing bonding agents, decreased lesion size, the results did not hold statistical significance (Paschos et al, 2009), and an in-vivo study by Leizer et al in 2010 concluded that application of Pro Seal had neither clinical nor statistical significance in preventing lesions when compared with a control. Conversely, another research paper concluded that Light Bond, an unfilled sealant, and Pro Seal, a filled sealant, were both significant in reducing demineralization in-vivo with Pro Seal providing a higher level of protection from lesion progression compared with controls (Shinaishin et al, 2011). Studies by Hu and Featherstone in 2005, Salar et al in 2007, Buren et al in 2008, and Behnan et al in 2010 all came to the similar conclusion that Pro Seal significantly prevented demineralization during orthodontic treatment. In addition to its ability to release fluoride ions, Pro Seal is also able to be recharged when acidulated phosphate fluoride introduces fluoride ions into the environment (Soliman et al, 2006).

The resolution of WSLs post orthodontic treatment has been evaluated in literature. A 2004 study followed patients 12 and 26 weeks after completion of treatment and found that lesions had decreased by a third and a half, respectively, without any special intervention (Willmot, 2004). Another study in 2010 by Shungin et al confirmed these findings in a 12-year follow-up, showing that white-spot decrease was most significant after 1 year, and, although pre-orthodontic levels were never reached, they continued to decrease even 12 years after debonding (Shungin et al, 2010). Decrease in WSLs after debonding can be attributed to several factors including tooth surface 
abrasion during tooth brushing, removal of the etiologic factor of plaque adhesion on orthodontic brackets, and the normal process of remineralization (Shungin et al, 2010).

\section{Significance and Purpose}

This study will serve as a pilot study and attempt to add to the evidence already present regarding Pro Seal and investigate Opal Seal's effectiveness in preventing demineralization. Currently, the bonding protocol at the University of Louisville Orthodontic Clinic allows for use of the resident's choice of bonding agents, whether it be Assure (Reliance Orthodontic Products), Pro Seal, or Opal Seal, and the majority of comprehensive orthodontic cases are bonded using Assure. The results of this study will be used to reevaluate bonding protocol in the orthodontic clinic and perhaps establish greater use of a particular bonding agent if one proves to be statistically significant in its prevention of demineralization.

\section{Hypotheses}

Null hypothesis:

Neither Pro Seal nor Opal Seal provide a statistically significant decrease in demineralization compared to Assure through the first 6 months of orthodontic treatment.

\section{Alternative hypothesis:}

Pro Seal and/or Opal Seal provide a statistically significant decrease in demineralization compared to Assure through the first 6 months of orthodontic treatment. 


\section{CHAPTER II}

\section{METHODS AND MATERIALS}

\section{IRB Approval}

Permission to conduct this study was granted by the Institutional Review Board of the University of Louisville on March 13, 2012. IRB tracking \#: 11.0666.

\section{Study Design and Sample}

This study was a retrospective chart review and the study population consisted of 26 consecutive patients who began comprehensive orthodontic treatment at the University of Louisville Orthodontic Clinic during the months of August 2011 and September 2011. The 26 patients were distributed as follows: 9 patients bonded using Assure, 8 patients bonded using Pro Seal, and 9 patients bonded using Opal Seal. Orthodontic treatment was initiated and provided by the six first year residents who bonded approved treatment planned patients according to standard clinic protocol and who were also responsible for documenting treatment progress with intraoral photographs. Clinical bonding protocol is as follows:

1) Pumice every tooth to be bonded with a slow speed handpiece and prophy cup.

2) Isolate the dentition using a Nola appliance. 
3) Etch the entire labial surface of every tooth to be bonded with $37 \%$ phosphoric acid.

4) Apply Assure, Pro Seal, or Opal Seal to entire labial surface of etched teeth and light cure material.

5) Bond brackets to teeth with light-cure adhesive.

6) Review standardized oral hygiene instructions with patient and guardian.

Standard pretreatment and six-month progress intraoral photographs were used to evaluate number of decalcification surfaces. Six teeth - maxillary right canine to maxillary left canine - were evaluated on each patient using the pretreatment and sixmonth progress photographs, except in those cases where certain teeth were excluded for reasons such as tooth missing, tooth not initially bonded with specific study group bonding agent, or poor photographic quality.

Each tooth included in the study was assigned a number of demineralization areas designated by WSLs at pretreatment and at six-month progress, with the highest possible of surfaces being four per tooth - mesial, distal, incisal, and gingival. The percentage increase in WSLs was calculated among the three groups, and statistical analysis was performed to determine whether there was any statistically significant benefit, and, possibly more important, a clinically significant benefit, of Pro Seal or Opal Seal in reducing the incidence of demineralization compared with the Assure control group. 


\section{Inclusion/Exclusion Criteria}

Only patients who began comprehensive treatment during the months of August 2011 and September 2011 were eligible to be included in the study. The following were the criteria used to exclude patients from the study:

1. Patients undergoing limited orthodontic treatment.

2. Patients under the age of 10.

3. Patients with physical or mental handicaps.

\section{Data Collection}

The current protocol at the University of Louisville Orthodontic Clinic is to take a standard set of intraoral photographs as a part of pretreatment records and also at sixmonths to document treatment progress. The number of tooth surfaces with WSLs was identified at pretreatment and at six-month progress for each patient using the right buccal, frontal, and left buccal intraoral photographs. Each patient included in the study had a maximum of six teeth evaluated - maxillary right canine, maxillary right lateral incisor, maxillary right central incisor, maxillary left central incisor, maxillary left lateral incisor, and maxillary left canine. In some patients one or more teeth were excluded for one of the following reasons:

1. The tooth/teeth did not have a bracket bonded at the initial appointment.

2. The tooth/teeth were bonded using a different bonding agent than the bonding agent for that particular group.

3. The tooth/teeth had a bracket that needed to be removed and rebonded for any reason during the first 6 months of comprehensive treatment. 
Each tooth was divided into four surfaces - mesial, distal, incisal, and gingival so the minimum number of surfaces with WSLs per tooth was 0 and the maximum number of surfaces with WSLs per tooth was 4 at each time point. The WSL increase from pretreatment to six-month progress was then calculated for each patient in preparation to run statistical tests to determine the effectiveness of each bonding agent. The photographs were each interpreted twice by a single provider, with the second reading occurring five days after the initial reading, in an effort to evaluate consistency and accuracy.

\section{Statistical Analysis}

For categorical characteristics, the frequency of the characteristic between the different bonding agent groups was compared and tested using Pearson's chi-squared test. For continuous characteristics, the one-way ANOVA or Kruskal-Wallis test was used to test for differences between the bonding agent groups. For the association of two continuous characteristics, the Spearman and Pearson's correlation coefficient were used. 


\section{CHAPTER III}

\section{RESULTS}

\section{Data Description}

The data consisted of 3 groups of 26 subjects, 9 of which used Assure, 9 of which used Opal Seal, and 8 of which used Pro Seal bonding agents. There were 20 female and 6 male subjects, and they ranged in age from 11 to 50 years. However, subjects for which it was impossible to experience a change in score (i.e. where initial decalcification surfaces $=$ total tooth surfaces, IS=TTS) were eliminated from analysis. The sample size using this criteria was 24 subjects, 19 of which were female and 5 of which were male. This criteria also effectively reduced the Pro Seal sample size from 8 to 6 .

Table 1 gives distribution of subject characteristics by bonding agent group, including age, sex, and also PS-IS (progress decalcification surfaces minus initial decalcification surfaces).

\section{Statistical Analysis}

For categorical characteristics, the frequency of the characteristic between the different bonding agent groups was compared and tested using Pearson's chi-squared test. For continuous characteristics, the one-way ANOVA or Kruskal-Wallis test was used to test for differences between the bonding agent groups. For the association of two continuous characteristics, the Spearman and Pearson's correlation coefficient was used. 
The response variable PS-IS was treated as a numeric covariate. Alternatively, since there were only 4 unique values for this quantity, analysis was conducted using the following transformations for the response variable:

1) Using the proportion PS-IS divided by the amount of change possible from IS, which is TTS-IS.

2) Using the arcsin square-root transformation for the above.

3) Using logistic regression, with PS-IS as the number of events, and TTS-IS as the number of trials (the number of opportunities for decalcification of tooth surfaces considering all tooth surfaces that had not yet been decalcified).

Logistic regression has the advantage that it increases the sample size, since it includes each tooth surface that has not been decalcified as a unit that can experience an event. The number of opportunities for an event in this study is then 463 .

\section{Results}

Primary Analysis: There was no statistically definitive evidence of a difference in change in surface (PS-IS) between the three bonding agents ( $\mathrm{p}$-value $=0.35$ using untransformed response, $\mathrm{p}=0.57$ using the proportion as response, $\mathrm{p}=0.56$ using $\arcsin$ squareroot transformation, and $\mathrm{p}=0.41$ using logistic regression). However, because of the small sample size more attention should be given to confidence intervals than pvalues. For this reason Table 3a displays Odds Ratios (OR's), Log OR's, and $95 \%$ Confidence Intervals for Opal Seal and Pro Seal groups, using the Assure Control group as the reference. One can see that the OR's for both Opal Seal and Pro Seal are less than 1 , and, equivalently, that the log OR's for both are negative, indicating a trend toward 
reduction in odds or probability of a tooth surface becoming decalcified for both groups compared to Assure Control. Since the OR's are less than 1, the log OR's and 95\% Cl's for $\log$ OR's are a more intuitive representation of this reduction in change in score compared to Assure Control. The log OR for Pro Seal is -1.34, and the 95\% Cl's are -3.4 and 0.77 respectively. This represents a possibly large significant reduction in odds of a tooth surface becoming decalcified. This could warrant a further larger confirmation study. These results are consistent with Figure 1, which displays boxplots for PS-IS for each group. The Opal Seal and Pro Seal groups have boxplot with means closer to 0 than the Assure Control group (the diamonds in the boxplots represent the means in each group). The boxplot for Pro Seal compared to Assure Control is particularly noteworthy. Table $3 \mathrm{~b}$ displays the same quantities as $3 \mathrm{a}$, but for age and gender. As before, tooth surface decalcification is the response. The odds ratio of 0.61 for age means that for every increase of 1 year in age the estimated odds of a tooth surface becoming decalcified is 0.61 compared to the year before. In other words, the estimated probability of a tooth surface becoming decalcified decreases with age. The odds ratio of 0.75 for females compared to males for tooth surface decalcification mean that the odds of a tooth surface becoming decalcified for a female is 0.75 compared to the odds of tooth surface becoming decalcified for a male. This is consistent with Figure 2, which shows boxplot for PS-IS for males and females.

Figure 1 shows that identifier \#1 is an outlier in this analysis. This is because this is the only subject with a PS minus IS equal to 4. The next highest value for PS-IS is 2 . However when this subject is omitted from the analysis, the p-value increases from 0.35 to 0.49 . There was also no evidence of association of gender with change in surface 
measurement ( $\mathrm{p}$-values range from 0.83 to 0.67 depending on transformation used, 0.28 using exact Wilcoxon, and 0.16 using exact Wilcoxon with Subject \#1 removed).

The (negative) association with age and tooth surface decalcification is closer to significant depending on which analysis is used. The p-value is 0.072 using logistic regression, and 0.034 using logistic regression with Subject \#1 removed.

More attention should be paid to analysis using logistic regression in this case, since it takes into account the fact that change in tooth surface measurement cannot be negative (a tooth surface cannot go from decalcified to calcified), and it models the opportunities for tooth surface decalcification, as well as the number of tooth surfaces actually experiencing decalcification. Compared to other methods, logistic regression uses more of the available information.

It should be kept in mind that this is a small observational study, and therefore lack of significant $\mathrm{p}$-values $(\mathrm{p} \leq 0.05)$ do not signify or prove that there is no difference or association between bonding agent and change in surface measurement. Descriptive statistics such as means, medians and confidence intervals (such as those shown in Table 1 through Table 3, and shown graphically in Figures 1-3) should be given more weight than p-values in these situations due to the inability of small sample sizes to provide statistical power. 
Table 1: Distribution of Patient Characteristics With Respect to Group

\begin{tabular}{|c|c|c|c|c|c|}
\hline \multirow[b]{2}{*}{ Variables } & \multicolumn{4}{|c|}{ Group } & \multirow[b]{2}{*}{ P Value } \\
\hline & $\begin{array}{c}\text { Total } \\
(\mathrm{N}=26)\end{array}$ & $\begin{array}{l}\text { Assure } \\
\text { Control } \\
(\mathrm{N}=9)\end{array}$ & $\begin{array}{c}\text { Opal Seal } \\
(\mathrm{N}=9)\end{array}$ & $\begin{array}{c}\text { Pro Seal } \\
(\mathbf{N}=8)\end{array}$ & \\
\hline Sex & & & & & $1.000^{\dagger}$ \\
\hline $\mathrm{F}(\%)$ & $20(76.9)$ & 7 (77.8) & 7 (77.8) & $6(75.0)$ & \\
\hline $\mathrm{M}(\%)$ & $6(23.1)$ & $2(22.2)$ & $2(22.2)$ & $2(25.0)$ & \\
\hline Age & & & & & 0.540 \\
\hline $\mathrm{N}$ & 26 & 9 & 9 & 8 & \\
\hline Mean $(95 \% \mathrm{CI})$ & $\begin{array}{c}15.7(12.8- \\
18.7)\end{array}$ & $\begin{array}{c}13.7(13.2- \\
14.1)\end{array}$ & $\begin{array}{c}17.8(9.7- \\
25.8)\end{array}$ & $\begin{array}{c}15.8(12.3- \\
19.2)\end{array}$ & \\
\hline $\begin{array}{l}\text { Median (min - } \\
\max )\end{array}$ & $\begin{array}{c}14.0(11.0- \\
50.0)\end{array}$ & $\begin{array}{c}14.0(13.0- \\
15.0)\end{array}$ & $\begin{array}{c}13.0(11.0- \\
50.0)\end{array}$ & $\begin{array}{c}14.5(11.0- \\
27.0)\end{array}$ & \\
\hline PS - IS & & & & & 0.470 \\
\hline $\mathrm{N}^{1}$ & 24 & 9 & 9 & 6 & $0.346^{*}$ \\
\hline Mean $(95 \% \mathrm{CI})$ & $\begin{array}{c}0.5(0.1- \\
0.9)\end{array}$ & $\begin{array}{c}0.8(-0.1- \\
1.6)\end{array}$ & $\begin{array}{c}0.4(-0.0- \\
0.9)\end{array}$ & $\begin{array}{c}0.2(-0.2- \\
0.5)\end{array}$ & $0.563 * *$ \\
\hline $\begin{array}{l}\text { Median (min - } \\
\max )\end{array}$ & $\begin{array}{c}0.0(0.0- \\
4.0)\end{array}$ & $\begin{array}{c}0.0(0.0- \\
4.0)\end{array}$ & $\begin{array}{l}0.0(0.0- \\
2.0)\end{array}$ & $\begin{array}{c}0.0(0.0- \\
1.0)\end{array}$ & $\begin{array}{c}0.197 * * \\
*\end{array}$ \\
\hline \multicolumn{6}{|c|}{$\begin{array}{l}{ }^{\dagger} \text { Exact test } \\
{ }^{*} \text { Kruskal-Wallis Test; } * * \text { arcsin square root transform } ;{ }^{* * *} \text { logistic regression } ; 1 \text { - only subjects with TTS not equal to IS } \\
\text { included for PP-IS } \\
\text { test using proportion }-0.56 \text {. }\end{array}$} \\
\hline
\end{tabular}


Table 2: Distribution of Demographic Characteristics With Respect to PS-IS

\begin{tabular}{|c|c|c|c|c|c|c|c|}
\hline \multirow{3}{*}{$\begin{array}{l}\text { Variables } \\
\text { Sex }\end{array}$} & \multicolumn{5}{|c|}{ PS - IS } & & \\
\hline & $\begin{array}{c}\text { Total } \\
(\mathrm{N}=24)\end{array}$ & $\begin{array}{c}0.0 \\
(\mathrm{~N}=16)\end{array}$ & $\begin{array}{c}1.0 \\
(\mathrm{~N}=6)\end{array}$ & $\begin{array}{c}2.0 \\
(\mathrm{~N}=1)\end{array}$ & $\begin{array}{c}4.0 \\
(\mathrm{~N}=1)\end{array}$ & \multicolumn{2}{|c|}{ P Values } \\
\hline & & & & & & 0.79 & $0.83 *$ \\
\hline $\mathrm{F}(\%)$ & $19(79.2)$ & $14(87.5)$ & $3(50.0)$ & $1(100.0)$ & $1(100.0)$ & $0.68 * *$ & $0.67 * * *$ \\
\hline $\mathrm{M}(\%)$ & $5(20.8)$ & $2(12.5)$ & $3(50.0)$ & $0(0.0)$ & $0(0.0)$ & $0.26^{4}$ & $0.28^{5}$ \\
\hline Age & & & & & & 0.38 & $0.37 *$ \\
\hline Mean(SD) & $16.0(7.9)$ & $17.3(9.4)$ & $13.7(1.4)$ & 11 & 14 & $0.275 * *$ & $0.072 * * *$ \\
\hline $\begin{array}{l}\text { Median(Min- } \\
\text { Max) }\end{array}$ & $14(11-50)$ & $14(13-50)$ & $13.5(12-16)$ & 11 & 14 & $0.034^{4}$ & $0.50^{5}$ \\
\hline \multicolumn{6}{|c|}{$\begin{array}{l}\text { * Using proportion; } * * \text { arcsign square root; } * * * \text { logistic regression; } 4 \text { logistic regression with } \\
\text { identifier } 1 \text { eliminated; } 5 \text { - exact Wilcoxon; - exact Wilcoxon with id=1 removed for Sex }-0.16 \\
\text { Output - Only Subjects with IS not equal to TTS included }\end{array}$} & & \\
\hline
\end{tabular}

Table 3a: Odds Ratios, Log Odds Ratios, and 95\% Confidence Intervals (Response-change in score for a tooth surface that was not initially decalcified, either 0 or 1 ).

\begin{tabular}{|c|c|c|c|c|c|c|c|}
\hline & \multirow[b]{2}{*}{ OR } & \multicolumn{2}{|c|}{$95 \% \mathrm{CI}$} & \multirow[b]{2}{*}{ Log OR } & \multicolumn{2}{|c|}{$95 \% \mathrm{CI}$} & \multirow[b]{2}{*}{$\mathrm{P}$} \\
\hline & & Lower & Upper & & Lower & Upper & \\
\hline Assure Control & - & - & - & - & - & - & - \\
\hline OpalSeal & 0.734 & 0.211 & 2.554 & -0.309 & -1.556 & 0.938 & 0.620 \\
\hline ProSeal & 0.261 & 0.032 & 2.154 & -1.343 & -3.442 & 0.767 & 0.260 \\
\hline
\end{tabular}

Assure Control used as Reference; Abbreviations -OR- Odds Ratio; Log OR- log of the Odds Ratio.

Table 3b: Odds Ratios, Log Odds Ratios, and 95\% Confidence Intervals for Age and Gender

(Response - change in score for a tooth surface that was not initially decalcified, either 0 or 1 ).

\begin{tabular}{|c|c|c|c|c|c|c|c|}
\hline & \multirow[b]{2}{*}{ OR } & \multicolumn{2}{|c|}{$95 \% \mathrm{CI}$} & \multirow[b]{2}{*}{ Log OR } & \multicolumn{2}{|c|}{$95 \%$ CI } & \multirow[b]{2}{*}{$\mathrm{P}$} \\
\hline & & Lower & Upper & & Lower & Upper & \\
\hline Age & 0.608 & 0.354 & 1.045 & -0.216 & -0.451 & 0.019 & 0.072 \\
\hline Gender* & 0.748 & 0.198 & 2.818 & -0.126 & -0.703 & 0.450 & 0.668 \\
\hline
\end{tabular}

*Female vs. Male - Male is reference level; Abbreviations -OR- Odds Ratio; Log OR- log of the Odds Ratio. 


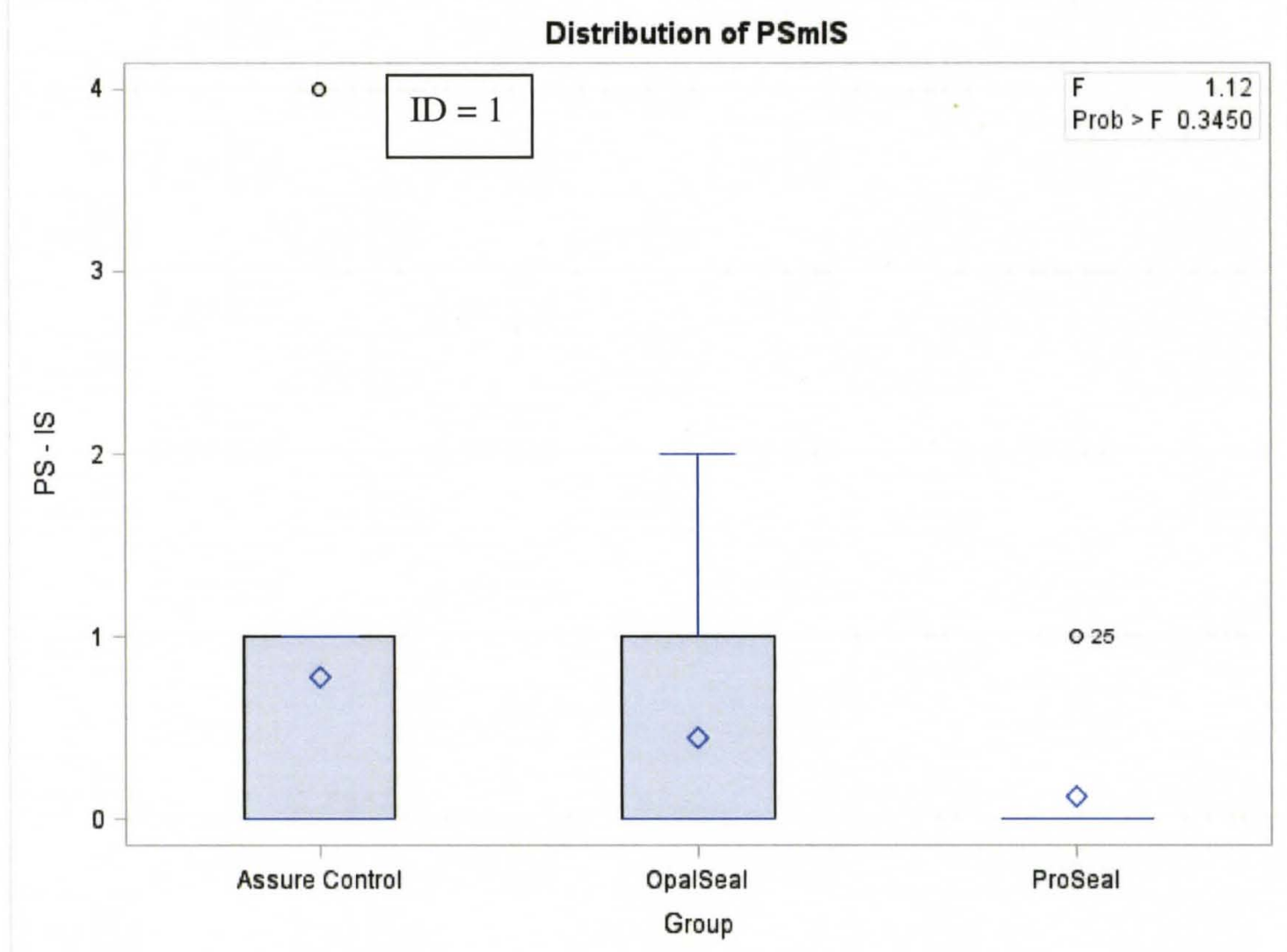

Figure 1: Boxplots of PS-IS for the three different bonding agents. Kruskall-Wallis and ANOVA p-values almost identical. 


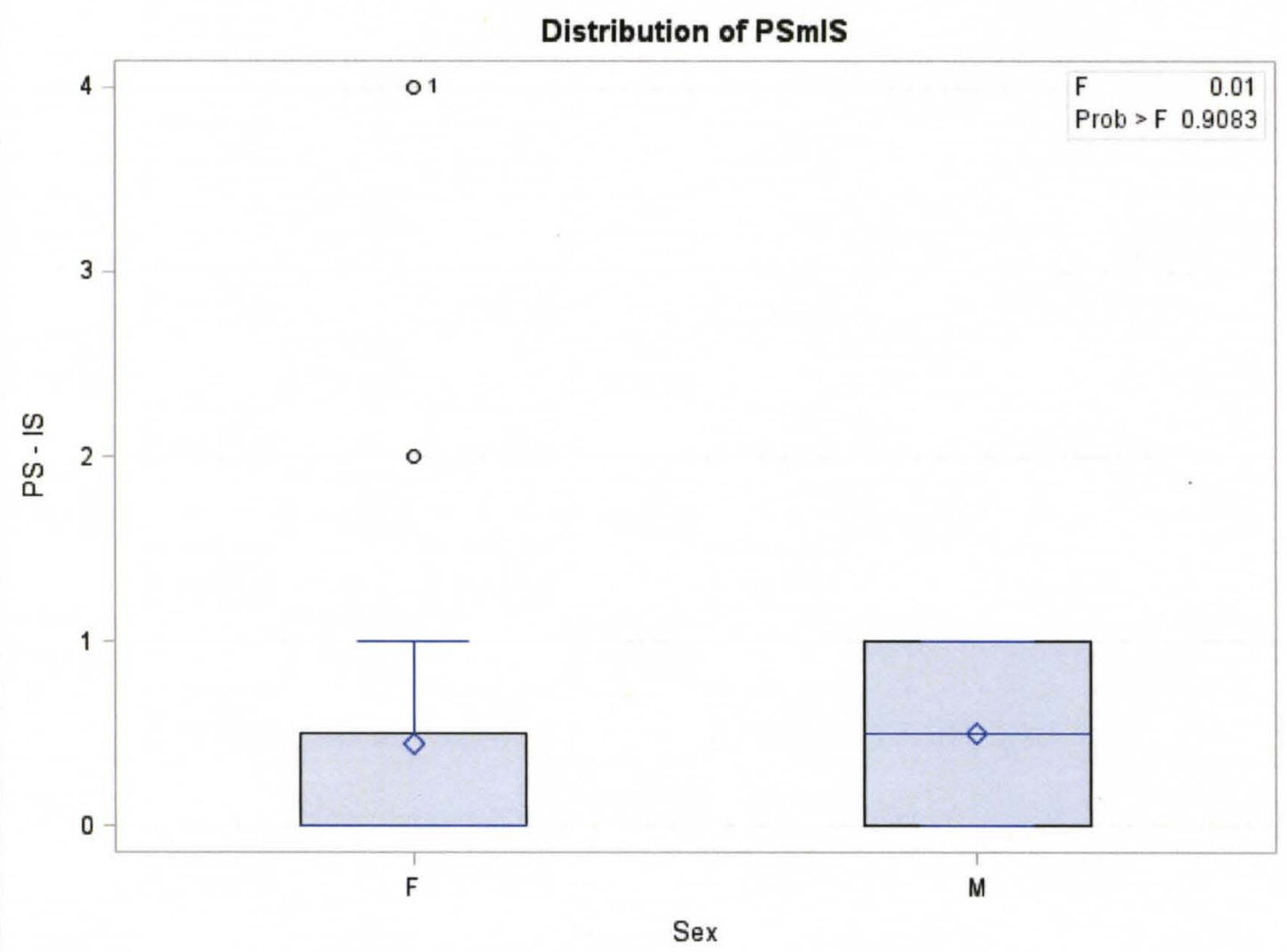

Figure 2: Boxplots of PS-IS by Gender t-test $\mathrm{p}=0.91$; Wilcoxon $\mathrm{p}=0.39(\mathrm{p}=0.25$ when $\mathrm{id}=1$ is removed). 
Fit Plot for PSmIS

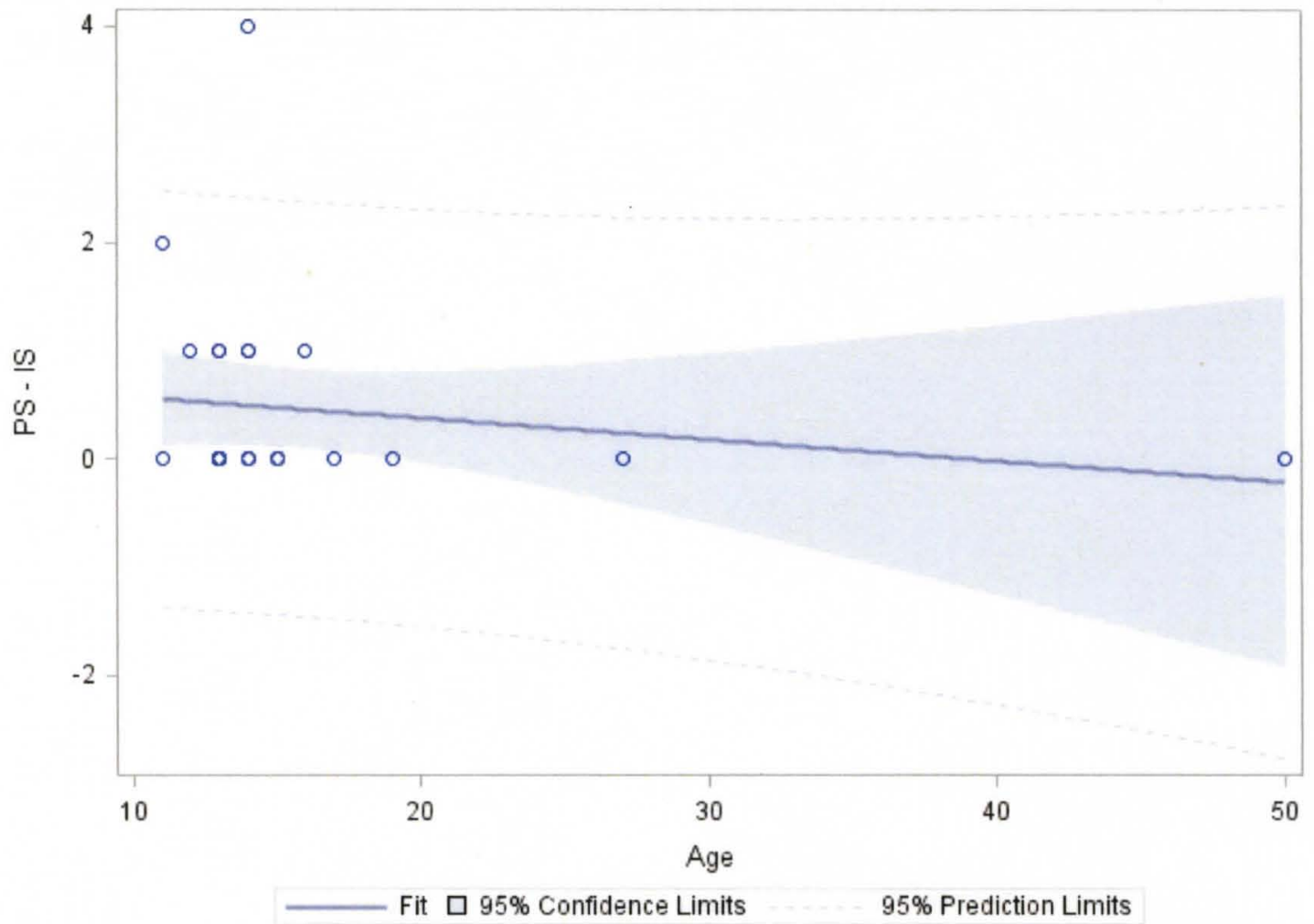

Figure 3: Scatter plot and fit for PS-IS and Age: Spearmen correlation coefficient-0.23, $\mathrm{p}=0.27$; Pearson correlation coefficient: $-0.17, \mathrm{p}=0.42$. With $\mathrm{ID}=1$ removed, Spearmen correlation coefficient $=-0.28, \mathrm{p}=0.18$; Pearson correlation coefficient: $-0.22, \mathrm{p}=0.30$. 


\section{CHAPTER IV}

\section{DISCUSSION}

With this serving as a pilot study, increased sample size and longer follow-up will be instrumental in building upon the data in upcoming years. Even though statistically speaking there was no significant different in demineralization rates between Assure, Pro Seal, and Opal Seal, this was a small observational study, and therefore lack of significant $\mathrm{p}$-values $(\mathrm{p} \leq 0.05)$ do not signify or prove that there is no difference or association between bonding agent and demineralization rates. Descriptive statistics such as means, medians and confidence intervals (such as those shown in Table 1, and shown graphically in Figures 1-3) should be given more weight than p-values in these situations. With this in mind, the data suggests that Pro Seal may prevent demineralization better than Opal Seal and Assure with Opal Seal performing better than Assure. There also appears to be a negative correlation between demineralization rates and age.

As the results of are evaluated, there are a several things that should be noted. Although every effort was made to produce the most objective data possible, this was a pilot study and many improvements could be made in future studies to increase its strength and validity.

There are several different methods of quantifying demineralization including, but not limited to, clinical evaluation, photograph evaluation, polarized light microscopy, 
atomic force microscopy, confocal laser scanning microscopy, and light-induced fluorescence. Of all these methods, photograph evaluation is likely the least scientific and least objective; however, it was chosen for this study due to the lack of access to the more sophisticated methods and the relative ease with which photographs could be taken and assessed. Photographic evaluation is subject to a wide variety of errors and subjectivity especially when variables such as lighting and camera settings are not standardized. If standard photography is continued to be utilized in future studies rather than the one of the aforementioned methods of quantifying demineralization, it would be advisable to standardize lighting, camera model, focal length, and camera settings such as aperture and shutter speed. A wide variety in the quality of photographs was noted in this study, so treatment providers should also be trained more extensively in the art of proper intraoral photography. Another shortcoming of this study was that the design only allowed for analysis of the quantity of demineralization during treatment and not the quality of it. Many studies in the past utilizing photographs have used the ordinal scale developed by Gorelick et al that described the extent of cavitation present; however, it was impossible to quantify the demineralization in that manner due to limited information the photographs in this study afforded.

Even if every effort is made to standardize intraoral photographs, utilization of photographs to detect and demineralization is not an exact science as false positives and false negatives are possible with human evaluation of photographs. In an effort to keep these false readings to a minimum, the photographs in this study were each interpreted twice, with the second reading occurring five days after the initial reading, to ensure accuracy and both readings were found to be consistent. If the photographic evaluation 
method is continued in future studies, it may be prudent to have the photographs interpreted by several practitioners to further reduce any bias.

Since the six-month progress photographs were taken with the orthodontic appliances in place, the identification of decalcification areas was significantly more difficult than if the appliances had been removed and teeth properly cleaned prior to obtaining photographs. Many of the intraoral photographs were unusable from a clinical diagnosis perspective due to the presence of plaque or other buildup around the brackets, gingival margins, and other surfaces of the teeth. Although removing orthodontic appliances introduces additional complexities, doing so would help add to the strength of futures studies by allowing areas of demineralization to be more easily and consistently identified. At the very least, a proper cleaning should be implemented prior to taking photographs even if the fixed appliances are not removed.

In addition to the nuances of the photography itself, variability in the rendering of treatment by different treatment providers may also prevent absolute control over treatment results. Although standardized instructions were written and given orally to the providers, it was impossible to monitor every provider to ascertain that proper protocol was followed. In future studies more effort should be made to monitor treatment to assure that protocols are followed and also to ensure that clinical notes are accurate.

With this serving as a pilot study, increased sample size and longer follow-up will be instrumental in providing better information about the effects Opal Seal and Pro Seal have on demineralization during comprehensive patient treatment at the University of Louisville Orthodontic Clinic. Regardless of advances in technology and efforts to 
prevent demineralization during orthodontic treatment, the best prevention appears to still be patient education both prior to and during fixed appliance orthodontic treatment. 


\title{
CHAPTER V
}

\section{CONCLUSION}

\begin{abstract}
Although this study did not show any statistically significant evidence that Pro Seal or Opal Seal prevents demineralization more effectively than Assure, this was a small pilot study and lack of significant p-values do not prove or signify that there is no difference or association between bonding agent and demineralization rates. However, descriptive statistics suggest that Pro Seal may most effectively prevent demineralization compared with Opal Seal and Assure. Further studies with increased sample size and longer follow-up are needed to provide stronger statistical and clinical information.
\end{abstract}




\section{REFERENCES}

Behnan SM, Arruda AO, Gonzalez-Cabezas C, Sohn W, Peters MC. In-vitro evaluation of various treatments to prevent demineralization next to orthodontic brackets. Am J Orthod Dentofacial Orthop 2010;138:712e1-712e7.

Benham AW, Campbell PM, Buschang PH. Effectiveness of pit and fissure sealants in Reducing white spot lesions during orthodontic treatment. Angle Orthod 2009;79: 337-344.

Buren JL, Staley RN, Wefel J, Qian F. Inhibition of enamel demineralization by an enamel sealant, Pro Seal: an in-vitro study. Am J Orthod Dentofacial Orthop 2008;133:S88-94.

Chadwick BL, Roy J, Knox J, Treasure ET. The effect of topical fluorides on decalcification in patients with fixed orthodontic appliances: a systematic review. Am J Orthod Dentofacial Orthop 2005;128:601-6.

Chang HS, Walsh LJ, Freer TJ. Enamel demineralization during orthodontic treatment. Aetiology and prevention. Australian Dental Journal 1997;42(5):322-7.

Farhadian N, Miresmaeili A, Eslami B, Mehrabi S. Effect of fluoride varnish on enamel demineralization around brackets: an in-vivo study. Am J Orthod Dentofacial Orthop 2008;133:S95-8.

Frazier MC, Southard TE, Doster PM. Prevention of enamel demineralization during orthodontic treatment: an in vitro study using pit and fissure sealants. Am J Orthod Dentofacial Orthop 1996;110-459-465.

Gorelick L, Geiger AM, Gwinnett AJ. Incidence of white spot formation after bonding and banding. Am J Orthod Dentofacial Orthop 1982;81(2):93-98.

Heintz S, Georg P. Oral health for the orthodontic patients. Philadelphia: Mosby 1999; p.2-9.

Hess E, Campbell PM, Honeyman AL, Buschang PH. Determinants of enamel decalcification during simulated orthodontic treatment. Angle Orthod 2011;81:836-842. 
$\mathrm{Hu} \mathrm{W}$ and Featherstone JDB. Prevention of enamel demineralization: an in-vitro study using light-cured filled sealant. Am J Orthod Dentofacial Orthop 2005;128:592600 .

Leizer C, Weinstein M, Borislow AJ, Braitman LE. Efficacy of a filled-resin sealant in preventing decalcification during orthodontic treatment. Am J Orthod Dentofacial Orthop 2010;137:796-800.

Lovrov S, Hertrich K, Hirschfelder U. Enamel demineralization during fixed orthodontic treatment - incidence and correlation to various oral-hygiene parameters. J Orofac Orthop 2007;68:353-363.

Mitchell L. Decalcification during orthodontic treatment with fixed appliances - an overview. Br J Orthod 1992;19:199-205.

Paschos E et al. Effect of different bonding agents on prevention of enamel demineralization around orthodontic brackets. Am J Orthod Dentofacial Orthop 2009;135:603-612.

Rogers S, Chadwick BL, Treasure ET. Fluoride-containing orthodontic adhesives and decalcification in patients with fixed appliances: a systematic review. Am J Orthod Dentofacial Orthop 2010;138:390.e1-390.e8.

Salar DV, Garcia-Godoy F, Flaitz CM, Hicks MJ. Potential inhibition of demineralization in vitro by fluoride-releasing sealants. JADA 2007;138(4):502-506.

Shinaishin SF, Ghobashy SA, EL-Bialy TH. Efficacy of light-activated sealant on enamel Demineralization in orthodontic patients: an atomic force microscope evaluation. The Open Dentistry Journal 2011;5:179-186.

Shungin D, Olsson AI, Persson M. Orthodontic treatment-related white spot lesions: a 14-year prospective quantitative follow-up, including bonding material assessment. Am J Orthod Dentofacial Orthop 2010;138:136.e1-136.e8.

Soliman MM, Bishara SE, Wefel J, Heilman J, Warren JJ. Fluoride release rate from an orthodontic sealant and its clinical implications. Angle Orthod 2006;76:282-288.

Trimpeneers LM and Dermaut LR. A clinical evaluation of the effectiveness of a fluoride-releasing visible light-activated bonding system to reduce demineralization around orthodontic brackets. Am J Orthod Dentofacial Orthop 1996;110:218-222.

Willmot DR. White lesions after orthodontic treatment: does low fluoride make a difference? Journal of Orthodontics 2004;31:235-242. 


\section{CURRICULUM VITAE \\ Robin Jae Choi}

103 South Chadwick Road, Apt \#2

Louisville, KY 40222

email: robinjchoi@gmail.com

phone: (240) 462-6171

\section{EDUCATION}

University of Louisville Dental School

Department of Orthodontics

Louisville, KY

Certificate of Orthodontics, expected June 2012

Masters in Oral Biology, expected August 2012

University of Maryland, Baltimore

Baltimore College of Dental Surgery

Baltimore, MD

Doctor of Dental Surgery, Magna Cum Laude, May 2010

Class Rank: $1 / 123$ years $1-3$, top $5 \%$ at graduation

University of Maryland, College Park

Robert H. Smith School of Business

College Park, MD

University Honors Program and Citation

Bachelor of Science in Finance, Bachelor of Science in Accounting

Double Degree, Magna Cum Laude, May 2003

GPA: 3.92/4.00, Class Rank: top 5\%

\section{HONORS AND AWARDS}

Magna Cum Laude, Certificate of Merit, 2010

University of Maryland Baltimore College of Dental Surgery

Honor in recognition of graduating within the top $5 \%$ of dental class.

Omicron Kappa Upsilon National Dental Honor Society, 2010

University of Maryland Baltimore College of Dental Surgery

Invite and induction limited to top $12 \%$ of graduating class demonstrating excellence in scholarship, exemplary traits of character and qualities for future professional growth and attainments. 
Gorgas Odontological Honor Society, 2008

University of Maryland Baltimore College of Dental Surgery

Invite for membership to students whose overall academic performance ranks in top $1 / 3$ of class.

Gamma Pi Delta Prosthodontic Honor Society, 2008

University of Maryland Baltimore College of Dental Surgery

Invite for membership to students with high scholastic achievement in the field of prosthetic dentistry.

American Dental Association Foundation Dental Student Scholarship, 2008

American Dental Association

One of 25 students selected nationwide on academic performance during first year dental school.

Dr. Charles W. Hennessey Memorial Scholarship, 2007

University of Maryland Baltimore College of Dental Surgery

Honors one student demonstrating high academic standing after the first year of dental school.

University of Maryland Baltimore Diversity Scholarship, 2006 - 2010

University of Maryland Baltimore College of Dental Surgery

Award to incoming student for academic merit prior to matriculation.

Magna Cum Laude, 2003

University of Maryland, College Park, Robert H. Smith School of Business

Honor reserved for the top $5 \%$ of graduating class

Graduated with 3.92 cumulative GPA, 4.0 Finance GPA, 4.0 Accounting GPA

Office of Multi-Ethnic Student Education Asian American Academic Excellence Award, 2003

University of Maryland, College Park, Robert H. Smith School of Business

Awarded to one graduating student with exceptional academic achievement.

Ernst \& Young Education Excellence Fund Scholarship, 2002

University of Maryland, College Park, Robert H. Smith School of Business

Scholarship granted to accounting major based on merit and dedication to the field of accounting.

University of Maryland Honors Scholarship, 2001

University of Maryland, College Park, Robert H. Smith School of Business

Merit based award for students in the University Honors Program with high academic performance.

University of Maryland Dean's Scholarship, 1999

University of Maryland, College Park, Robert H. Smith School of Business 
University award for incoming first-year students based on merit.

University of Maryland Alumni Association Scholarship, 1999

University of Maryland, College Park, Robert H. Smith School of Business

One of two recipients among incoming class selected for demonstrating academic excellence.

Maryland Distinguished Scholar Award, 1999

The State of Maryland

Scholarship for Maryland residents exhibiting superior academic achievement.

\section{PROFESSIONAL EXPERIENCE}

Audit \& Assurance Services

Deloitte \& Touche LLP

Tysons Corner, VA

September 2003 - April 2004, June 2002 - August 2002

Collaborated with audit teams to gather evidential matter and perform substantive tests designed to verify client transactions and ensure proper valuation and documentation in accordance with Generally Accepted Accounting Principles.

Financial Services Intern

UBS PaineWebber

Largo, MD

June 2001 - September 2001

Responsible for assisting in maintaining existing client relationships and prospecting for new clients, conducted Bloomberg and Internet research and analysis of target companies for Financial Advisors and performed administrative tasks to support office operations.

Biological Research Intern

United States Department of Defense, Uniformed Services University

Bethesda, MD

June 1999 - August 1999, June 1998 - August 1998

Performed team research to examine immune system response to Traumatic Brain Injuries (TBI), assisted physicians during experimental surgeries and responsible For documenting results.

\section{PROFESSIONAL AND SCHOLASTIC ORGANIZATIONS}

American Association of Orthodontists, 2010 - present

Omicron Kappa Upsilon, 2010 - present

Gorgas Odontological Honor Society (secretary), University of Maryland Dental School, $2008-2010$

Gamma Pi Delta Prosthodontic Honor Society, University of Maryland Dental School, $2008-2010$

Committee on Curriculum Innovation, University of Maryland Dental School, 2008 $-2010$ 